\title{
HOW DO THEY ADD UP? THE INTERACTION BETWEEN THE PLACEBO AND TREATMENT EFFECT : A SYSTEMATIC REVIEW.
}

Rémy Boussageon ${ }^{1}$, Jeremy Howick ${ }^{2}$, Raphael Baron ${ }^{3}$, florian naudet ${ }^{4}$, bruno falissard $^{5}$, Ghina Harika-Germaneau ${ }^{6}$, Issa Wassouf ${ }^{6}$, François Gueyffier ${ }^{7}$, Nemat Jaafari ${ }^{6}$, and Clara Blanchard $^{8}$

${ }^{1}$ Université Claude Bernard Lyon 1

${ }^{2}$ University of Oxford

${ }^{3}$ Universite Lyon 1 Faculte de Medecine Lyon-Est

${ }^{4}$ University Hospital Centre Rennes

${ }^{5}$ INSERM

${ }^{6}$ Centre Hospitalier Henri Laborit

${ }^{7}$ Centre Hospitalier Universitaire de Lyon

${ }^{8}$ University of Poitiers

October 2, 2021

\begin{abstract}
Aim: The placebo effect and the specific effect are often thought to add up (additive model). Whether this is true or whether there is an interaction between the two, can modify the external validity of a trial. This assumption of additivity was tested by Kleijnen et al. in 1994 but the data produced since then has not been synthetized. In this review, we aimed to systematically review the literature to determine whether additivity held. Methods: We searched Medline and Psychinfo up to 10/01/2019. Studies using the balanced placebo design (BPD), testing two different strengths of placebos, were included. The presence of interaction was evaluated by comparing each group in BPD with analysis of variance or covariance. Results: 30 studies were included and the overall risk of bias was high: four found evidence of additivity and 16 studies found evidence of interaction (seven had evidence of positive additivity). Conclusion: Evidence of additivity between placebo and specific features of treatments was rare in our sample. For ailments that are placebo-responsive, pragmatic trials should be preferred to increase their external validity.
\end{abstract}

\section{Hosted file}

Manuscript_BJCP.docx available at https://authorea.com/users/439089/articles/540140-how-dothey-add-up-the-interaction-between-the-placebo-and-treatment-effect-a-systematic-review 